\title{
RIGIDITY OF HOLOMORPHIC MAPPINGS AND A NEW SCHWARZ LEMMA AT THE BOUNDARY
}

\author{
DANIEL M. BURNS AND STEVEN G. KRANTZ
}

\section{INTRODUCTION}

The uniqueness portion of the classical Schwarz lemma has played an interesting historical role. Usually first encountered in the classification of the conformal self-maps of the disc, it arises in more general considerations of automorphism groups, in the construction of the Carathéodory and Kobayashi/Royden metrics, and in a variety of contexts in analysis on manifolds. Useful references for this material are [AHL, KR1, KOB, GK1, GK2, YAU].

Generalizations of the disc Schwarz lemma to multiply-connected domains in one complex variable are generated naturally and easily using the uniformization theorem. The generalization to several complex variables requires insights of a different nature. For example, the so-called Carathéodory-Cartan-Kaup-Wu theorem [WU] says in part that if $\Omega$ is a bounded domain in $\mathbb{C}^{n}$ and if $\Phi$ is a holomorphic self-map of $\Omega$ that fixes a point $P \in \Omega$, then the holomorphic Jacobian determinant det $\operatorname{Jac} \Phi(P)$ has modulus less than or equal to 1 , and equals 1 if and only if $\Phi$ is a biholomorphism of $\Omega$. An interesting and important feature of this result is that it has a global hypothesis (that $\Omega$ be mapped to itself) and a local hypothesis (the condition on the behavior of the mapping at $P$ ). The conclusion is then a strong global one. We will see this paradigm repeated in the work that follows.

The purpose of this paper is to seek versions of the last stated result when the point $P$ lies in the boundary of $\Omega$. (A primitive version of such a result appears in [KR2].) This problem, while of considerable intrinsic interest, is also related to a variety of other work in the literature. We now indicate some of these connections.

Our interest in this problem arose originally from a question of Warren Wogen: Does there exist a holomorphic self-mapping $\Phi$ of the ball $B$ in $\mathbb{C}^{2}$ (i) which takes the point $\mathbf{1}=(1,0) \in \partial B$ to itself (in a suitable sense) and (ii) for which the set $\partial(\Phi(B))$ has "high order of contact", as a set, with the boundary $\partial B$ of the target ball? Here part of the problem is to find a suitable interpretation for the phrase "high order of contact". The mappings

Received by the editors May 3, 1993.

1991 Mathematics Subject Classification. Primary 32H15, 32H20, 32H99.

Key words and phrases. Holomorphic mapping, boundary rigidity, Schwarz lemma.

The first author was supported in part by grant \#DMS9004149 from the National Science Foundation. The second author was supported in part by grants \#DMS8800523 and \#DMS9101104 from the National Science Foundation. 
$\Phi_{\varepsilon}\left(z_{1}, z_{2}\right)=\left((1-\varepsilon) z_{1}+\varepsilon,(1-\varepsilon) z_{2}\right), \varepsilon>0$, satisfy (i) and (ii) with the boundary of the image ball having order of contact 1 with the boundary of the target ball (the tangent planes agree but the second fundamental forms do not). The mapping $\Phi_{0}$ is the identity and the order of contact is infinite. Wogen's question may be interpreted as asking whether there are maps which are intermediate to the $\Phi_{\varepsilon}, \varepsilon>0$, and id $=\Phi_{0}$. The original interest in constructing such maps was in finding counterexamples to certain assertions about composition operators (see [WOG]).

As our understanding of the problem developed, we also saw that it relates to a result of $H$. Alexander [ALE]: If $U \cap B$ and $U^{\prime} \cap B$ are boundary neighborhoods in the ball and if $\Phi$ is a biholomorphic mapping of these neighborhoods which extends $C^{2}$ to the boundary, then $\Phi$ must be the restriction to $U \cap B$ of a biholomorphism of the entire ball. S. Pincuk [PIN] generalized this result to bounded strictly pseudoconvex domains with real analytic boundaries and $\mathrm{W}$. Rudin [RU1] reduced the hypothesis of $C^{2}$ to the boundary to an assumption which is even weaker than continuity at a point. One interpretation of the main result of the present paper is that the hypothesis of direct coincidence of boundary neighborhoods in the results of Alexander, Pincuk, and Rudin may be weakened to high order of contact of image boundary and target boundary. (See also [GK3] for other more general versions of the Alexander phenomenon-in particular, that paper characterizes not just the biholomorphic mappings of the ball but mappings which are "approximately" biholomorphic mappings of the ball.)

The organization of this paper is as follows: Section 2 presents a boundary uniqueness result on the disc. The relationship of this result to the questions just discussed is not immediately apparent, but is clarified later. Section 3 shows how to extend the result of Section 2 to the ball in $\mathbb{C}^{n}$. Section 4 shows how to use variants of the Fornæss Imbedding Theorem and the Lempert theory of extremal discs for the Kobayashi metric to derive a result for strictly pseudoconvex domains. Section 5 discusses a generalization of the Schwarz uniqueness theorems of the previous sections. Section 6 discusses geometric interpretations of the main results and returns to the original question of order of contact. The question of Wogen is then recalled and answered. Section 7 considers only very briefly analogous problems on weakly pseudoconvex domains.

\section{A RESULT ON THE DISC ABOUT BOUNDARY DERIVATIVES}

Let $D$ be the unit disc centered at $0 \in \mathbb{C}$.

Theorem 2.1. Let $\phi: D \rightarrow D$ be a holomorphic function from the disc to itself such that

$$
\phi(\zeta)=1+(\zeta-1)+O\left((\zeta-1)^{4}\right)
$$

as $\zeta \rightarrow 1$. Then $\phi(\zeta) \equiv \zeta$ on the disc.

Remark 1. Results such as this one appear in the literature of conformal mappings (see, for instance, [VEL]) with the additional hypothesis that $\phi$ be univalent (and often the function is assumed to be quite smooth-even analytic-in a neighborhood of 1 ). The theorem presented here has no such hypothesis; so 
far as we know it is new. Even more surprising is that the exponent 4 is sharp: simple geometric arguments show that the function

$$
\phi(\zeta)=\zeta-\frac{1}{10}(\zeta-1)^{3}
$$

satisfies the hypotheses of the theorem with 4 replaced by 3 . Note also in the proof that $O\left((z-1)^{4}\right)$ can be replaced by $o\left((z-1)^{3}\right)$. A similar remark applies to Sections 3,4 , and 5 below.

X. Huang, in the papers [HU1, HU2, HU3], has explored additional conditions under which the exponent in the error term may be decreased.

Proof of Theorem 2.1. Consider the holomorphic function

$$
g(\zeta)=\frac{1+\phi(\zeta)}{1-\phi(\zeta)}
$$

Then $g$ maps the disc $D$ to the right half plane. By the Herglotz representation (this is just an application of the Banach-Alaoglu theorem; see [AHL]), there must be a positive measure $\mu$ on the interval $[0,2 \pi)$ and an imaginary constant $\mathscr{C}$ such that

$$
g(\zeta)=\frac{1}{2 \pi} \int_{0}^{2 \pi} \frac{e^{i \theta}+\zeta}{e^{i \theta}-\zeta} d \mu(\theta)+\mathscr{C} .
$$

We use the hypothesis on $\phi$ to analyze the structure of $g$ and hence that of $\mu$. To wit,

$$
g(\zeta)=\frac{1+\zeta+O(\zeta-1)^{4}}{1-\zeta-O(\zeta-1)^{4}}=\frac{1+\zeta}{1-\zeta}+O(\zeta-1)^{2} .
$$

From this and equation $(*)$ we easily conclude that the measure $\mu$ has the form $\mu=\delta_{0}+\nu$, where $\delta_{0}$ is ( $2 \pi$ times) the Dirac mass at the origin and $\nu$ is another positive measure on $[0,2 \pi)$. In fact, a nice way to verify the positivity of $\nu$ is to use the equation

$$
\frac{1+\zeta}{1-\zeta}+O(\zeta-1)^{2}=\frac{1}{2 \pi} \int_{0}^{2 \pi} \frac{e^{i \theta}+\zeta}{e^{i \theta}-\zeta} d\left(\delta_{0}+\nu\right)(\theta)+\mathscr{C}
$$

to derive a Fourier-Stieltjes expansion of $\delta_{0}+\nu$ and then to apply the Herglotz criterion [KAT, p. 38].

We may simplify equation $(* *)$ to

$$
O(\zeta-1)^{2}=\frac{1}{2 \pi} \int_{0}^{2 \pi} \frac{e^{i \theta}+\zeta}{e^{i \theta}-\zeta} d \nu(\theta)+\mathscr{C} .
$$

Pass to the real part of the last equation, thus eliminating the constant $\mathscr{C}$. Since $\nu$ is a positive measure, we thus see that the real part of the integral on the right of this last equation represents a positive harmonic function $h$ on the disc that satisfies

$$
h(\zeta)=O(\zeta-1)^{2} .
$$

In particular, $h$ takes a minimum at the point $\zeta=1$ and is $O\left(|z-1|^{2}\right)$ there as well. This contradicts Hopf's lemma [KR1] unless $h \equiv 0$. But $h \equiv 0$ 
implies that $\nu \equiv 0$, hence that

$$
g(\zeta)=\frac{1+\zeta}{1-\zeta}
$$

Therefore $\phi(\zeta) \equiv \zeta$, and the theorem is proved.

Remark 2. Notice that the formulation of Theorem 2.1 is a boundary uniqueness theorem in the vein of the uniqueness part of the classical Schwarz lemma. However, the result sidesteps the original questions about "order of contact". In fact the Riemann Mapping Theorem guarantees that, with any reasonable definition of the phrase, there are univalent holomorphic maps of the disc to the disc so that the image boundary has arbitrarily high (even infinite order) contact with the target boundary. Thus Theorem 2.1 addresses some more rigid structural phenomenon.

We defer our consideration of the original geometric question to $\S 6$.

\section{A RESULT FOR THE BALL}

Theorem 3.1. Let $B \subset \mathbb{C}^{n}$ be the unit ball. Let $\Phi: B \rightarrow B$ be a holomorphic mapping of the ball to itself such that

$$
\boldsymbol{\Phi}(z)=1+(z-1)+O\left(|z-1|^{4}\right)
$$

as $z \rightarrow \mathbf{1}$. (Here $\mathbf{1}$ denotes the distinguished boundary point $\mathbf{1}=(1,0, \ldots, 0)$ of the ball.) Then $\phi(z) \equiv z$ on the ball.

Proof. There is no useful Herglotz representation on the ball (however, see [AIZ] for related ideas): this is a deep fact which cannot be circumvented. Thus we present a new argument that reduces the ball case to the disc case. For simplicity we restrict attention to dimension two.

For each point $a \in B$ let $\mathscr{L}_{a}$ be the complex line joining $a$ and 1 . Let $d_{a}$ be the complex disc given by $\mathscr{L}_{a} \cap B$. Now for fixed $a$ consider the holomorphic function

$$
\begin{aligned}
\psi & : D \longrightarrow B \\
& \zeta \longmapsto(\zeta, 0) .
\end{aligned}
$$

Define also the mapping $\phi_{a}: B \longrightarrow B$, which is an automorphism of the ball mapping $d_{0}$ onto $d_{a}$ and fixing 1 . (That such maps exist follows from elementary geometric considerations, or see [RU2] for an explicit formula.) Finally define

and

$$
\begin{aligned}
& \pi_{1}: B \longrightarrow B, \\
& \pi_{1}:\left(z_{1}, z_{2}\right) \longmapsto\left(z_{1}, 0\right) ;
\end{aligned}
$$

$$
\begin{aligned}
& \eta: d_{0} \longrightarrow D, \\
& \eta:\left(z_{1}, 0\right) \longmapsto z_{1} .
\end{aligned}
$$

Then the function

$$
\begin{aligned}
H_{a}: & D \longrightarrow D \\
& \zeta \longmapsto \eta \circ \pi_{1} \circ\left(\phi_{a}\right)^{-1} \circ \Phi \circ \phi_{a} \circ \psi(\zeta)
\end{aligned}
$$


is well defined. Moreover, it is straightforward to check that $H$ satisfies the hypotheses of Theorem 2.1 (since $\Phi$ agrees with the identity to high order, the composition $\left(\phi_{a}\right)^{-1} \circ \Phi \circ \phi_{a}$ is the identity to high order). It follows that $H_{a}(\zeta) \equiv \zeta$.

Now set

$$
G_{a}=\left(\phi_{a}\right)^{-1} \circ \Phi \circ \phi_{a} \circ \psi(\zeta) \equiv\left(g_{a}^{1}(\zeta), g_{a}^{2}(\zeta)\right) .
$$

The statement that $H_{a}(\zeta) \equiv \zeta$ means that $g_{a}^{1}(\zeta) \equiv \zeta$. But

$$
\left|g_{a}^{1}(\zeta)\right|^{2}+\left|g_{a}^{2}(\zeta)\right|^{2}<1
$$

for $\zeta \in D$. Letting $|\zeta| \rightarrow 1$ yields then that $\left|g_{a}^{2}(\zeta)\right| \rightarrow 0$. Thus $g_{a}^{2} \equiv 0$. It now follows that the image of $G_{a}$ already lies in $d_{0}$. As a result, it must be that $\Phi$ preserves $d_{a}$. But this can only hold for every choice of $a$ if $\Phi$ is the identity mapping.

The proof is now complete.

\section{Generalization of the Result to strongly CONVEX AND TO STRONGLY PSEUDOCONVEX DOMAINS}

It is clear that the methods of the last section will not apply to strongly pseudoconvex domains. Indeed most strongly pseudoconvex domains have no automorphisms except the identity (see [GK1]), so we are missing a major tool.

However, an inspection of the proof of Theorem 3.1 shows that the principal geometric construct is of a family of mappings of the disc into the ball such that the image analytic discs are holomorphic retracts of the entire domain (this is where the global nature of the result, alluded to in the introduction, comes in). Thanks to work of Lempert [LEM1], we know a large family of domains for which such special analytic discs exist:

Proposition 4.1 (Lempert). Let $\Omega \subset \mathbb{C}^{n}$ be a smoothly bounded, strictly convex (in the real sense) domain with $\mathscr{C}^{k}$-boundary $(k \geq 6)$. Let $Q \in \Omega$ and $P \in \partial \Omega$. Then there exist unique holomorphic mappings

$$
\varphi_{P, Q}: \bar{D} \longrightarrow \bar{\Omega}, \quad \psi_{P, Q}: \bar{\Omega} \longrightarrow \bar{D}
$$

which are $\mathscr{C}^{k-4}$ up to the boundary, and such that:

(1) $\varphi_{P, Q}(0)=Q, \varphi_{P, Q}(1)=P$;

(2) $\psi_{P, Q} \circ \varphi_{P, Q}(\zeta)=\zeta$, for every $\zeta \in \bar{D}$;

(3) if $\varphi: D \rightarrow \Omega$ is holomorphic, and if $\psi_{P, Q} \circ \varphi(\zeta)=\zeta$ for $\zeta \in D$, then $\varphi(\zeta) \equiv \varphi_{P, Q}(\zeta)$

Note that (2) implies that $\varphi_{P, Q}$ is extremal for the infinitesimal Kobayashi metric on $\Omega$ in the direction $\xi:=\varphi_{P, Q}^{\prime}(0) \in T_{Q}(\Omega)$, while $\psi_{P, Q}$ is the extremal for the "dual" infinitesimal Carathéodory metric at $\xi$.

Proof. The statements of the proposition follow from [LEM1, Théorème 3] and [LEM2].

Given Proposition 4.1, the proof of Theorem 3.1 gives the following result. 
Theorem 4.2. Let $\Omega$ be a smoothly bounded, strictly convex domain in $\mathbb{C}^{n}$ with $\mathscr{C}^{6}$ boundary, and let $P \in \partial \Omega$ be fixed. If $\Phi: \Omega \rightarrow \Omega$ is a holomorphic mapping such that

$$
\Phi(z)=z+O\left(|z-P|^{4}\right)
$$

then $\Phi(z) \equiv z$.

Proof. Indeed, the maps $\varphi_{P, Q}$ above replace $\phi_{a} \circ \psi$ in the proof of Theorem 3.1 , and the $\psi_{P, Q}$ play the role of $\eta \circ \pi_{1} \circ\left(\phi_{a}\right)^{-1}$ there. The uniqueness argument at the end of the proof of Theorem 3.1 is here achieved by (3) of Proposition 4.1.

Unfortunately, Lempert's results fail for a general strictly pseudoconvex domain (see [SIB]), but they remain true in a neighborhood of $P$, in directions approximately $\mathbb{C}$-tangent to $\partial \Omega$ at $P$. More precisely, we have the following.

Proposition 4.3. Let $\Omega$ be a smoothly bounded, strongly pseudoconvex domain in $\mathbb{C}^{n}$ with $\mathscr{C}^{6}$ boundary, and let $P \in \partial \Omega$ be fuxed. Then there exists $Q \in \Omega$, an open neighborhood $V \subset \Omega$ of $Q$, and holomorphic mappings

$$
\varphi_{P, Q^{\prime}}: \bar{D} \longrightarrow \Omega, \quad \psi_{P, Q^{\prime}}: \Omega \longrightarrow \bar{D}
$$

as in Proposition 4.1 above, for every $Q^{\prime} \in V$. Furthermore, given any neighborhood $U \subset \mathbb{C}^{n}$ of $P$, one can assume that both $Q$ and the images $\varphi_{P, Q^{\prime}}(\bar{D})$ lie in $\bar{\Omega} \cap U$.

Proof. Given $U$, that the stationary disc $\varphi_{P, Q}$ exists for some $Q \in U \cap \Omega$ is shown in [LEM1, p. 468]. The existence of the open set $V \ni Q$, and of the corresponding $\varphi_{P, Q^{\prime}}, Q^{\prime} \in V$, follows from [LEM1, Proposition 10, p. 446]. To get the dual functions $\psi_{P, Q^{\prime}}$, we need the following preparatory geometrical lemma.

Lemma 4.4. Let $\Omega$ and $P$ be as in Proposition 4.3 above. Then there exist a neighborhood $\Omega^{\prime}$ of $\Omega$, a bounded, strictly convex domain $\Omega^{\prime \prime} \subset \mathbb{C}^{n}$, and a holomorphic map $F: \Omega^{\prime} \rightarrow \mathbb{C}^{n}$ such that:

(1) for a suitable open neighborhood $W$ of $F(P), F$ is biholomorphic, $F: F^{-1}(W) \rightarrow W$

(2) $F(\Omega) \subset \Omega^{\prime \prime}$;

(3) $F(\partial \Omega) \cap W=\partial \Omega^{\prime \prime} \cap W$.

Admitting the lemma for the moment, we return to the proof of Proposition 4.3. We now choose the $Q$ and $V$ more carefully, namely so that $V \subset U \cap$ $F^{-1}(W) \cap \bar{\Omega}$, where $W, F$ are as in the lemma. Then the mappings $F \circ \varphi_{P, Q^{\prime}}$ are stationary discs for the strictly convex domain $\Omega^{\prime \prime} \subset \mathbb{C}^{n}$. Thus there exist dual mappings

$$
\psi_{F(P), F\left(Q^{\prime}\right)}^{0}: \bar{\Omega}^{\prime \prime} \longrightarrow \bar{D}
$$

and the mappings $\psi_{P, Q^{\prime}}=\psi_{F(P), F\left(Q^{\prime}\right)}^{0} \circ F$ will have all the desired properties in the statement of Proposition 4.3, as follows directly from Proposition 4.1.

Using Proposition 4.3, the proof of Theorem 4.2 extends directly to give: 
Theorem 4.5. Let $\Omega$ be a connected, smoothly bounded, strongly pseudoconvex domain. Let $P \in \partial \Omega$, and let $\Phi: \Omega \rightarrow \Omega$ be a holomorphic map such that $\Phi(z)=z+O\left(|z-P|^{4}\right)$. Then $\Phi(z) \equiv z$.

Proof. Find $Q, V$ as in Proposition 4.3 above. The proof as in Theorems 3.1 and 4.2 shows that $\Phi$ is the identity on each disc $\varphi_{P, Q^{\prime}}(D)$; in particular, $\Phi\left(Q^{\prime}\right)=Q^{\prime}$ for all $Q^{\prime} \in V$. By analytic continuation, $\Phi(z) \equiv z$.

We finish this section with the proof of Lemma 4.4. We start with Fornæss's embedding theorem [FOR], which gives a neighborhood $\Omega^{\prime}$ of $\Omega$, a holomorphic embedding $G: \Omega^{\prime} \hookrightarrow \mathbb{C}^{N}$ (for some large $N$ ), a smoothly bounded strictly convex set $\Omega_{0}^{\prime \prime} \subset \mathbb{C}^{N}$ such that $\partial \Omega_{0}^{\prime \prime}$ intersects $G\left(\Omega^{\prime}\right)$ transversally, and $G(\Omega)=\Omega_{0}^{\prime \prime} \cap G\left(\Omega^{\prime}\right)$. Let $T$ be the tangent space to $G\left(\Omega^{\prime}\right)$ at $G(P) ; T$ is isomorphic to $\mathbb{C}^{n}$, say $E: T \stackrel{\sim}{\longrightarrow} \mathbb{C}^{n}$ linearly. Let $\Pi: \mathbb{C}^{N} \rightarrow T$ be the Hermitian orthogonal projection onto $T$ (with respect to the usual euclidean metric on $\mathbb{C}^{N}$ ), so that $\Pi$ is $\mathbb{C}$-linear. We claim that the composition

$$
F: \Omega^{\prime} \stackrel{G}{\longrightarrow} \mathbb{C}^{N} \stackrel{\Pi}{\longrightarrow} T \stackrel{E}{\longrightarrow} \mathbb{C}^{n}
$$

will be the desired map, if we shrink $\Omega^{\prime}$ about $\bar{\Omega}$ as necessary. To see this, notice first that property (1) of Lemma 4.4 follows from the strict convexity of $\Omega_{0}^{\prime \prime}$ and the fact that $G$ is an embedding. (We may be required to shrink $\Omega^{\prime}$ here.) It is an easy exercise in the implicit function theorem to see that, in a neighborhood of $P, F$ is a local diffeomorphism of $\partial \Omega$ onto a strictly convex hypersurface in $\mathbb{C}^{n}$. Since $E \circ \Pi$ is linear, the compact set $E \circ \Pi\left(\bar{\Omega}_{0}^{\prime \prime}\right):=\bar{\Omega}_{1}^{\prime \prime}$ is convex and $F(\Omega) \subset \Omega_{1}^{\prime \prime}$. It is again a simple exercise in the implicit function theorem to see that the boundary of $\Omega_{1}^{\prime \prime}$ is smooth and strictly convex near $F(P)$. Thus there is a closed euclidean ball $\bar{B}$ which is tangent to $\partial \Omega_{1}^{\prime \prime}$ at $F(P)$ and which contains $\bar{\Omega}_{1}^{\prime \prime}$. Translate $\bar{B}$ a distance $\varepsilon>0$ in the direction of the inward euclidean normal to $\partial \Omega_{1}^{\prime \prime}$ at $F(P)$, to a new ball called $\bar{B}_{\varepsilon}$. For $\varepsilon$ sufficiently small, let $H_{\varepsilon}$ denote the (real) convex hull of $\bar{B}_{\varepsilon} \cup F(\bar{\Omega})$ in $\mathbb{C}^{n}$, so that the boundary of $H_{\varepsilon}$ coincides with $F(\partial \Omega)$ in a neighborhood of $F(P)$. The domain $H_{\varepsilon}$ is convex, but not smooth and not strictly convex. We can enlarge $H_{\varepsilon}$ slightly to a smoothly bounded, strictly convex set $\Omega^{\prime \prime} \subset \mathbb{C}^{n}$ whose boundary still agrees with $F(\partial \Omega)$ in a neighborhood of $F(P)$. That this last is possible is clear from a picture, but is elementary and tedious to write down explicitly, so we omit this verification. The $\Omega^{\prime \prime}$ just constructed satisfies all the claims of Proposition 4.4.

We close this section with the remark that Lemma 4.4 is an attempt to reembed $\Omega$ in $\mathbb{C}^{n}$ as a strictly convex domain, which is certainly impossible. The point here is that $F$ is globally defined, and does re-embed $\Omega$ as a strictly convex domain, at least in a neighborhood of $P$.

\section{ANOTHER DIRECT EXTENSION}

For $P \neq Q$ it is interesting to consider the setting of Theorem 4.2 with the modified hypothesis that

$$
\Phi(z)=Q+\mu(z-P)+O\left(|z-P|^{4}\right) ;
$$


here $\mu$ is some local holomorphic transformation which takes the tangent plane to $\partial \Omega$ at $P$ to the tangent plane to $\partial \Omega$ at $Q$. Of course we cannot hope to conclude under these circumstances that $\Phi$ is the identity. To see what the correct conclusion might be, consider two special cases:

(1) If $\Omega$ is the ball, then $\Phi$ may be composed with a unitary rotation taking $Q$ to $P$. This reduces the situation to the result of $\S 3$. We conclude that $\Phi$ is a rotation.

(2) If $\Omega$ is strongly pseudoconvex and there is a biholomorphism $\tau$ of $\Omega$ whose extension to the boundary takes $Q$ to $P$ then $\tau \circ \Phi$ satisfies the hypotheses of Theorem 4.5. We conclude that $\Phi$ is a biholomorphism of $\Omega$.

A generic strongly pseudoconvex $\Omega$ has no nontrivial biholomorphisms (see [GK1]); so the second case above can be considered descriptive but not prescriptive. Moreover, for any positive integer $m$ it is a simple matter to construct a strongly pseudoconvex domain $\Omega$ with boundary points $P, Q$ such that $\partial \Omega$ near $Q$ is (after a rigid motion) a $2 m$-order perturbation of $\partial \Omega$ near $P$-thus, it can be arranged that a rigid motion of $\Omega$ maps $\Omega$ into $\Omega$, maps $P$ to $Q$, and agrees with the identity to order $2 m$, yet the mapping is not biholomorphic. In summary, a generalization of our results in the direction we have been discussing must take a more restrictive form. We have the following:

Theorem 5.1. Let $\Omega \subset \mathbb{C}^{n}$ be a smoothly bounded, strongly pseudoconvex domain with $C^{\omega}$ boundary. Suppose $P, Q \in \partial \Omega$ and $\Phi$ is a local biholomorphic equivalence of $\partial \Omega$ sending $P$ to $Q$. If $F: \Omega \rightarrow \Omega$ is a holomorphic map such that

$$
F(z)=\Phi(z)+O\left(|z-P|^{4}\right)
$$

near $P$, then $\Phi$ continues analytically to a biholomorphism of $\Omega$ and $F(z) \equiv$ $\Phi(z)$ on $\Omega$.

Proof. We first note that the localized argument in the proof of Theorem 4.5 can be applied to $\Phi^{-1} \circ F$ in a neighborhood of $P$ to show that $F \equiv \Phi$ near $P$ in $\Omega$. We would like to use analytic continuation now, but must distinguish between two cases.

(1) $\partial \Omega$ is not spherical, i.e., is nowhere locally CR-equivalent to $\partial \mathbb{B}^{n}$. In this case, the theorem of Kruzhilin-Vitushkin [VIT] says that the local holomorphic equivalence $\Phi$ can be analytically continued along arbitrary continuous curves in $\partial \Omega$, as a local biholomorphic equivalence sending $\partial \Omega$ to itself. By analytic continuation, then, we conclude that $F$ extends $C^{\omega}$ to $\partial \Omega$, and has nonvanishing Jacobian determinant. The mapping $F$ is therefore a biholomorphism, since it is a self-map of $\Omega$.

(2) $\partial \Omega$ is spherical, i.e., locally $\mathrm{CR}$-equivalent to $\partial \mathbb{B}^{n}$ at some (and hence every, by analytic continuation) point. Then by a result of [BU], the universal cover $\tilde{\Omega}$ is biholomorphic to $\mathbb{B}^{n}$. The universal covering $\tilde{\Omega}^{\prime}$ of a sufficiently small open neighborhood $\Omega^{\prime}$ of $\Omega$ is also realizable as an open set $\mathbb{B}^{n} C$ $\tilde{\Omega}^{\prime} \subset \mathbb{C}^{n}$, and we let $\tilde{P} \in \partial \mathbb{B}^{n}$ be an inverse image of $P$ for this extended covering map. The mapping $F$ lifts to $\tilde{F}: \mathbb{B}^{n} \rightarrow \mathbb{B}^{n}$, and what we have proved above now shows that in a neighborhood of $\tilde{P}, \tilde{F}$ gives a local biholomorphic equivalence of $\partial \mathbb{B}^{n}$ to itself. But it is known from [CHM] that such a local equivalence is the restriction of a global automorphism $\tilde{\Phi}$ of $\mathbb{B}^{n}$. By analytic 
continuation, $\tilde{F} \equiv \tilde{\Phi}$ on $\mathbb{B}^{n}$, and $\tilde{\Phi}$ induces the claimed analytic continuation of $\Phi$ to $\Omega$.

Remark 3. In conclusion, we note that these two cases behave differently with respect to analytic continuation of boundary equivalences, namely, the theorem of Kruzhilin-Vitushkin is false if $\partial \Omega$ is spherical. We do not know, incidentally, whether in general the analytic continuation given by the Kruzhilin-Vitushkin theorem is single valued.

\section{GEOMETRIC INTERPRETATIONS}

\section{AND RETURN TO THE QUESTION OF ORDER OF CONTACT}

An elegant theorem of Ian Graham [GRA] calculates the asymptotic boundary behavior of the infinitesimal Carathéodory and Kobayashi metrics near a strongly pseudoconvex boundary point. The work of Fefferman in [FEF] calculates the asymptotic boundary behavior of the infinitesimal Bergman metric near a strongly pseudoconvex boundary point. In both papers the proofs make it clear that if two strongly pseudoconvex domains have local defining functions which agree to fourth order at a strongly pseudoconvex boundary point $P$ then the Carathéodory (resp. Kobayashi, resp. Bergman) metric has local boundary behavior at $P$ which is the same for both domains. Thus we see that the hypotheses of Theorem 4.5 are precisely what are needed to guarantee that the Carathéodory (resp. Kobayashi, resp. Bergman) metric near the distinguished boundary point $P$ has asymptotic boundary behavior which is identical to that for the pullback metric (under the mapping $\Phi$ ) near $P$. To what extent can this reasoning be reversed? That is, if $\Phi$ is a self-map of $\Omega$, taking a boundary point $P$ to itself and such that $\Phi$ preserves asymptotically some invariant metric near $P$, then must $\Phi$ be an automorphism of $\Omega$ ?

Interestingly, the problem is more subtle than this discussion suggests. For, the fourth order contact that we have been discussing is enough to guarantee identical local behavior of the metrics (for instance, it is enough to pick up the first term of the asymptotic expansion for the Bergman kernel at $P$ ), but it does not suffice to capture the global information that is necessary for the truth of the theorems we have been considering. Thus, at this time we do not have a purely geometric method for proving the theorems of Sections 3 and 4.

Now let us return to our original question concerning "geometric order of contact" of the image of a mapping with its target boundary. To give a more natural geometrical notion of contact here for our problem we consider an anisotropic notion appropriate to the strong pseudoconvexity of our domains. Let $\Omega$ as before be a smoothly bounded strongly pseudoconvex domain, and fix $P \in \partial \Omega$. Let $\rho$ be a defining function for $\partial \Omega$ near $P$. Normalize the coordinate system at $P$ so that $z=0$ at $P$, the $z_{1}$-direction is the complex normal direction, and $z^{\prime}:=\left(z_{2}, \ldots, z_{n}\right)$ are the complex tangential directions. Define $\delta(z)=\left(\left|z_{1}\right|^{2}+\left|z^{\prime}\right|^{4}\right)^{1 / 4}$. We say that a function $W_{N}=W_{N}(z)$ is of weight $N$ at $P$ if $\left|W_{N}(z)\right| \leq C \delta(z)^{N}$ near $P$, for some $C>0$. Similarly, in analogy with Landau's notation, we will denote by $w_{N}(z)$ a function such that $\lim _{z \rightarrow 0}\left|w_{N}(z)\right| / \delta^{N}(z)=0$. These notions are independent of the coordinate normalizations. 
Now let $\Phi$ be a holomorphic map which is $\mathscr{C}^{k}$ on $\Omega$ near $P \in \partial \Omega, \Phi(\Omega) \subset$ $\Omega^{\prime}, \Phi(P)=Q \in \partial \Omega^{\prime}$, and let $\rho^{\prime}$ be a defining function for $\partial \Omega^{\prime}$ at $Q$.

Definition 6.1. The set $\Phi(\partial \Omega)$ is said to have geometric contact with $\partial \Omega^{\prime}$ of weight $N$ at $\Phi(P)=Q$ if

$$
\rho^{\prime} \circ \Phi=h \cdot \rho+w_{N}
$$

where $h$ is a $\mathscr{C}^{k-1}$ positive function on $\bar{\Omega}$ near $P$ and $w_{N}$ is as above.

Remark 4. In this definition, $k$ must be at least $N$ for the definition to make intrinsic sense. See below for some remarks on more efficient measures of differentiability here.

Now let us relate this geometric notion of contact with the analytic osculation discussed earlier.

Proposition 6.2. Let $\mathbb{B}^{n}$ be the unit ball in $\mathbb{C}^{n}, n \geq 2$, and $\Phi$ a holomorphic mapping, $\Phi: \mathbb{B}^{n} \rightarrow \mathbb{B}^{n}$. Suppose $\Phi$ is $\mathscr{C}^{6}$ to the boundary near $P \in \partial \mathbb{B}^{n}$ and $Q=\Phi(P) \in \partial \mathbb{B}^{n}$. If $\Phi\left(\partial \mathbb{B}^{n}\right)$ has geometric contact of weight 6 with $\partial \mathbb{B}^{n}$ at $Q$, then there is a global biholomorphism $\Psi: \mathbb{B}^{n} \rightarrow \mathbb{B}^{n}$ such that

$$
\Phi(z)=\Psi(z)+o\left(|z-P|^{3}\right)
$$

near $P$.

Remark 5. The Riemann Mapping Theorem shows that the proposition is false when $n=1$. Concerning the sharpness of the proposition, and of Theorem 3.1, see Example 6.3 below.

Proof of Proposition 6.2. Without loss of generality, by composing $\Phi$ with automorphisms of $\mathbb{B}^{n}$, we can assume that $P=Q=(1,0, \ldots, 0)$. Conjugating with a Cayley transform, we can also replace $\mathbb{B}^{n}$ with $\mathscr{U}_{n}=\left\{(z, w) \in \mathbb{C}^{n-1} \times \mathbb{C} \mid\right.$ $\left.\operatorname{Im} w>|z|^{2}\right\}$, and $P$ with 0 . The correspondence can be given explicitly by

$$
w=-i \frac{z_{1}-1}{z_{1}+1}, \quad z_{i}=\frac{z_{j+1}}{z_{1}+1}, \quad j=1, \ldots, n-1 .
$$

The coordinates $(z, w)$ at 0 are, suitably renumbered, normalized as in Definition 6.1, and the proof of the proposition is an examination of our hypothesis $(*)$ with $N=6$ in terms homogeneous with respect to the dilations $T_{\delta}:(z, w) \rightarrow\left(\delta z, \delta^{2} w\right), \delta>0$.

Again, composing with automorphisms of $\mathscr{U}_{n}$, we can assume without loss of generality that $\Phi$ has an expansion near 0 :

$$
\Phi(z, w)=(z+A(z, w), w+B(z, w))
$$

where

$$
\begin{aligned}
& A(z, w)=\sum_{\nu=2}^{N} a_{\nu}(z, w)+w(N+1) \\
& B(z, w)=\sum_{\nu=3}^{N} b_{\nu}(z, w)+w(N+1)
\end{aligned}
$$


Here, $a_{\nu}, b_{\nu}$ are homogeneous of degree $\nu$ for the $T_{\delta}$-dilations. Also $W(k)$, as distinct from $W_{k}$, denotes an error term in an obvious sense. Similarly, $h$ can be written

$$
h=1+\sum_{\nu=2}^{N} h_{\nu}+w(N+1) .
$$

We will use $N \leq 6$ in these expansions.

Let $a_{\nu_{0}}$ be the first nonvanishing term in $A$; then $(*)$ shows that $b_{\nu_{0}+1}$ is the first nonvanishing term of $B$. Thus

$$
\Phi(z, w)=\left(z+a_{\nu_{0}}+\text { h.w.t., } w+b_{\nu_{0}+1}+\text { h.w.t. }\right)
$$

where "h.w.t." is "higher weight terms" and $\nu_{0} \geq 2$. The part of equation (*) homogeneous of weight $\nu_{0}+1$ (where $\nu_{0}<N$ ), and with $P=Q=0$, $\rho=\rho^{1}=\operatorname{Im} w-|z|^{2}$, is just

$$
(*)_{\nu_{0}+1} \quad h_{\nu_{0}-1}\left(\operatorname{Im} w-|z|^{2}\right)=-2 \operatorname{Re}\left\langle a_{\nu_{0}}, z\right\rangle+b_{\nu_{0}+1}
$$

where, for $v, v^{\prime} \in \mathbb{C}^{n-1},\left\langle v, v^{\prime}\right\rangle=\sum_{i=1}^{n-1} v_{i} \bar{v}_{i}^{\prime}$.

Now the operation

$$
\left(a_{\nu_{0}}, b_{\nu_{0}+1}\right) \longmapsto-2 \operatorname{Re}\left\langle a_{\nu_{0}}, z\right\rangle+\operatorname{Im} b_{\nu_{0}+1},
$$

evaluated at $\operatorname{Im} w=|z|^{2}$, is the operator " $L$ " on formal power series that was defined in formula (2.6) of [CHM]. Equation $(*)_{\nu_{0}+1}$ says precisely that $L\left(a_{\nu_{0}}, b_{\nu_{0}+1}\right)=0$. We shall consider $\nu_{0}=2,3$ directly; the cases $\nu_{0}=4,5$, which we shall also need, will then follow from Lemma 2.1 of [CHM].

The case $\nu_{0}=2$. In this case we have

$$
\begin{aligned}
& h_{1}=\langle\alpha, z\rangle+\langle\bar{\alpha}, \bar{z}\rangle, \quad \text { some } \alpha \in \mathbb{C}^{n}, \\
& a_{2}=Q(z)+\beta w, \quad Q \text { quadratic in } z, \quad \beta \in \mathbb{C}^{n}, \\
& b_{3}=C(z)+w\langle z, g a\rangle, \quad C \text { cubic, }, \gamma \in \mathbb{C}^{n} .
\end{aligned}
$$

Then equation $(*)_{3}$ becomes

$$
\begin{aligned}
\left(\frac{\langle\alpha, z\rangle w}{2 i}-\frac{\langle\bar{\alpha}, \bar{z}\rangle w}{2 i}\right)-\left(\langle\alpha, z\rangle|z|^{2}+\langle\bar{\alpha}, \bar{z}\rangle|z|^{2}\right)+\left(\frac{\langle\bar{\alpha}, \bar{z}\rangle w}{2 i}-\frac{\langle\alpha, z\rangle \bar{w}}{2 i}\right) \\
=-(\langle Q(z), z\rangle+\overline{\langle Q(z), z\rangle})-(w\langle\beta, z\rangle+\overline{w\langle\beta, z\rangle}) \\
\quad+\left(\frac{C(z)}{2 i}-\frac{\overline{C(z)}}{2 i}\right)+\left(\frac{w\langle z, \gamma\rangle}{2 i}-\frac{\bar{w} \overline{\langle z, w\rangle}}{2 i}\right) .
\end{aligned}
$$

Comparing like monomials on either side (let us label these with the notation 
$(p, q))$, we find that

$$
\begin{gathered}
0=\frac{C(z)}{2 i} \text { or } C(z)=0 \\
\frac{w\langle z, \alpha\rangle}{2 i}=\frac{w\langle z, \gamma\rangle}{2 i} \text { or } \alpha=\gamma ; \\
-\langle z, \alpha\rangle\langle z, z\rangle=-\langle Q(z), z\rangle \text { or } Q(z)=\langle z, \alpha\rangle z ; \\
\frac{\langle\alpha, z\rangle w}{2 i}-\frac{\langle z, \alpha\rangle \bar{w}}{2 i}=-w\langle z, \alpha\rangle-\bar{w} \overline{\langle\beta, z\rangle} ;
\end{gathered}
$$

hence $\alpha=-2 i \beta$, some $\beta \in \mathbb{C}^{n}$.

Before proceeding, we recall that the automorphisms of $\mathscr{U}_{n}$ with $0 \in \partial \mathscr{U}_{n}$ a fixed point and normalized as in $(* *)$ have the form

$$
(z, w) \longmapsto\left(\frac{z+w \beta}{1-2 i\langle z, \beta\rangle+c w}, \frac{w}{1-2 i\langle z, \beta\rangle+c w}\right),
$$

where $\beta \in \mathbb{C}^{n}$ is arbitrary and $\operatorname{Im} c=-|\beta|^{2}$. The calculations performed above tell us that, after composing with one of these automorphisms of $\mathscr{U}_{n}$, we may assume that $(* *)$ holds with $\nu_{0} \geq 3$.

The case $\nu_{0}=3$. Now we have

$$
\begin{aligned}
& h_{2}=q(z)+\alpha w+\bar{\alpha} \bar{w}+\bar{q}(z), \quad q \text { quadratic, } \alpha \in \mathbb{C}^{n} ; \\
& a_{3}=C(z)+w L(z), \quad C \text { cubic, } L \text { linear } ; \\
& b_{4}=Q(z)+w S(z)+\gamma w^{2}, \quad Q \text { quartic, } S \text { quadratic, } j \in \mathbb{C} .
\end{aligned}
$$

Thus $(*)_{4}$ becomes

$$
\begin{gathered}
\operatorname{Im}\left(q w+\alpha w^{2}\right)-2 \operatorname{Re} q|z|^{2}-\operatorname{Im}(q \bar{w})-2 \operatorname{Re}\left(\alpha w|z|^{2}\right)-|w|^{2} \operatorname{Im} \alpha \\
=\operatorname{Im}\left(Q+w S+\gamma w^{2}\right)-2 \operatorname{Re}[\langle C(z), z\rangle+w\langle L(z), z\rangle] .
\end{gathered}
$$

Comparing like terms now yields

$$
\begin{array}{rr}
(4,0) & Q \equiv 0 ; \\
(3,0) & S \equiv q ; \\
(2,0) & \alpha=\gamma ; \\
(3,1) & C(z)=q(z) \cdot z ; \\
(1,1) & \operatorname{Im} \alpha=0 ;
\end{array}
$$

hence $q \equiv 0$ and $L(z)=\alpha z$.

Note that we have now that $q=S=0$ and $C=z \cdot q=0$. The upshot of our calculation is that the mapping $\Phi$ agrees in form (up to order $\nu_{0}$ ) with an automorphism of $\mathscr{U}_{n}$ that fixes 0 . Composing $\Phi$ with the inverse of said automorphism (as we are obviously free to do), we have that $\Phi$ satisfies (**) with $\nu_{0}=4$.

The cases $\nu_{0}=4,5$. Applying Lemma 2.1 of [CHM, p. 233], we find that

$$
\left(\alpha_{4}, b_{5}\right)=(0,0) \text { and }\left(\alpha_{5}, b_{6}\right)=0 \text {. }
$$


Thus we have proved that the geometric order of contact 6 implies that

$$
\Phi(z, w)=(z, w)+(\text { terms of homogeneity at least six }) .
$$

This is almost the desired result. In terms of the usual notion of order, this result says that there is a constant $\alpha \in \mathbb{C}$ such that, without loss of generality,

$$
\Phi(z, w)=\left(z+\alpha w^{3}, w\right)+O\left(|z-P|^{4}\right) .
$$

We can now use a global argument related to Theorem 3.1 above. Consider the mapping of the upper half plane $\mathscr{H}:=\{\operatorname{Im} w>0\}$ to itself given by $w \rightarrow w+B(0, w)$. By the above we know that $B(0, w)$ is $O\left(|w|^{4}\right)$ at $w=0$ and so by the conjugated form of the boundary Schwarz lemma, $B(0, w) \equiv 0$. Now consider the map of $D$ into $\mathscr{U}_{n}$ given by

$$
w \longrightarrow \Phi(0, w)=(A(0, w), w+B(0, w))=(A(0, w), w) .
$$

By the uniqueness argument at the end of the proof of Theorem 3.1, it follows that $A(0, w) \equiv 0$. Hence, the coefficient $\alpha$ above is 0 , which proves the proposition.

Remark 6. Of course we could assume above that $\Phi\left(\partial \mathbb{B}^{n}\right)$ had contact of weight $N \geq 7$, and the proposition would follow from purely local calculations. It would certainly not be sharp then.

In the above proposition, we can weaken considerably the notion of differentiability on $\Phi$ and $h$ as follows: Say that $\Phi$ is admissibly differentiable at $P$ of weight $N$, if in coordinates normalized at $P=0$ as above, there exists an approximation of $\mathscr{P}(z, w)=(A(z, w), B(z, w))$ where $A-\sum_{\nu=0}^{N-1} a_{\nu}(z, w)=$ $w_{N}(z, w)$ and $B-\sum_{\nu=0}^{N} b_{\nu}(z, w)=w_{N}(z, w)$. Here the approximations are to hold on admissible approach regions at 0 in the sense of Koranyi. Note that the $a_{\nu}, b_{\nu}$ are assumed to be $T_{\delta}$-homogeneous as in the proof above. One can similarly define nontangential differentiability of order $N$, using nontangential approach regions. The corresponding concepts for the real function $h$ we leave to the reader. Proposition 6.2 follows if $\Phi$ is admissibly differentiable of weight 6 and nontangentially differentiable of order 3 at $P$. Presumably one does not need all derivatives in $z$ of $\Phi$ up to order 6 for the validity of Proposition 6.2. We have chosen to work with the notion of contact expressed in terms of weight because of its naturality with respect to CR-geometry, as in [CHM]. Example 6.3 below shows that, with this measure of contact, Theorem 3.1 and Proposition 6.2 are sharp.

Example 6.3. We will construct self-maps of the Siegel domain $\mathscr{U}_{2}$ to itself which describe with some precision how sharp the above results are. We start from the Riemann Mapping Theorem in $\mathbb{C}$, and take $V$ to be a smoothly bounded, simply connected open set $\subset \mathscr{H}$ (the upper half plane in $\mathbb{C}$ ) such that $\bar{V} \cap \partial \mathscr{H}$ in $\mathbb{C}$ contains a neighborhood of 0 in the real axis. Let $\varphi: \mathscr{H} \rightarrow V$ be a Riemann mapping (i.e., biholomorphism). Since $\varphi$ is one-to-one, $\varphi^{\prime}(w) \neq 0$, for all $w \in \mathscr{H}$. Since $\mathscr{H}$ is simply connected, we can choose a branch of $\sqrt{\varphi^{\prime}(w)}$ globally on $\mathscr{H}$. By the Pick-Schwarz lemma, applied to the map $\varphi$, 
we know that

$$
\frac{\left|\varphi^{\prime}(w)\right|^{2}}{|\operatorname{Im} \varphi(w)|^{2}} \leq \frac{1}{|\operatorname{Im} w|^{2}}
$$

By (\#), the mapping of $\mathbb{C} \times \mathscr{H} \subset \mathbb{C}^{2}$ to $\mathbb{C}^{2}$ given by

$$
F:(z, w) \longrightarrow\left(\sqrt{\varphi^{\prime}(w)} z, \varphi(w)\right)
$$

sends $\mathscr{U}_{2}$ to itself. Without loss of generality, we may choose $\varphi$ in such a way that

$$
\varphi(w)=w+\lambda w^{3}+O\left(|w|^{4}\right),
$$

where $\lambda$ is real, $\lambda>0$. Then, in a neighborhood of 0 , we have

$$
F(z, w)=\left(z+\frac{3}{2} \lambda w^{2} z, w+\lambda w^{3}\right)+O\left(|(z, w)|^{4}\right) .
$$

Here we have chosen the branch of $\sqrt{\varphi^{\prime}(w)}$ which is 1 at $w=0$. For this map, $F\left(\partial \mathscr{U}_{2}\right)$ has contact of weight 5 but not weight 6 ; the case $\nu_{0}=5$ above breaks down here. This example (extended to $\mathbb{C}^{n}$ ) also shows that Theorem 3.1 is sharp for all $n$. Note that the map $F$ actually extends analytically past 0 .

It is enlightening to attempt to carry out the proof of Proposition 6.2 for $n=1$ to see that the coefficients of $\Phi$ are not determined by the contact equation, no matter what order of contact is assumed. Note also that the higher dimensional example above has contact of $F\left(\partial \mathscr{U}_{2}\right)$ with $\partial \mathscr{U}_{2}$ along the entire curve $(0, u), u=\operatorname{Re} w \in \mathbb{R}$, near $u=0$. The contact weight is sharp at all points of this curve.

\section{DisCUSSION OF THE WEAKLY PSEUDOCONVEX CASE AND CLOSING REMARKS}

The analysis we have been discussing does not carry over to the case of weakly pseudoconvex domains. A simple example serves to illustrate the wealth of questions and problems available: Consider the domains

$$
\Omega_{m}=\left\{\left(z_{1}, z_{2}\right):\left|z_{1}\right|^{2}+\left|z_{2}\right|^{2 m}<1\right\},
$$

for $m$ a positive integer. What conditions near $1=(1,0)$ on a holomorphic mapping $\Phi: \Omega_{m} \rightarrow \Omega_{m}$ will force $\Omega$ to be the identity mapping? The key observation is that $\Omega_{m}$ covers the ball $B$ via the mapping $\psi_{m}\left(z_{1}, z_{2}\right)=$ $\left(z_{1},\left(z_{2}\right)^{m}\right)$. For any $\alpha=\left(\alpha_{1}, \alpha_{2}\right) \in B$ with $\alpha_{2} \neq 0$, the analytic disc $d_{\alpha}$ (see §3) lifts to $m$ extremal discs $\hat{d}_{\hat{\alpha}, j}, j=1, \ldots, m$, in $\Omega_{m}$. Then the analysis of $\S 3$ may be carried out with these extremal discs-provided that the mapping $\Phi$ has Taylor jet at $P$ which is sufficiently restricted so that after it is pushed down with the function $\psi_{m}$ off the branch locus, it satisfies the conditions of Theorem 3.1. Note in particular that the correct condition on the Taylor jet will be nonisotropic and will depend on $m$. X. Huang [HU1, HU2, HU3] has explored these matters in some detail. 
An argument similar to the one just outlined may be carried out near any weakly pseudoconvex boundary point that locally "covers" a strongly pseudoconvex boundary point. This is in fact a rather restrictive class, and has recently been described by Barletta and Bedford [BABE] (however, see also [GAV]).

\section{ACKNOWLEDGMENT}

We thank John Bland and Robert E. Greene for helpful discussions about the general nature of this problem and $\mathrm{Al}$ Baernstein for a useful conversation regarding the proof of Theorem 2.1. We also thank J. Moser and N. Sibony for their timely interest and suggestions.

\section{REFERENCES}

[AHL] L. Ahlfors, Conformal invariants, McGraw-Hill, New York, 1973.

[ALE] H. Alexander, Holomorphic mappings from the ball and polydisc, Math. Ann. 209 (1974), 249-256.

[AIZ] L. A. Aizenberg, Multidimensional analogues of the Carleman formula with integration over boundary sets of maximal dimension, Akad. Nauk. SSSR Sibirsk. Otdel. Inst. Fiz., Krasnoyarsk, 1985, pp. 12-22, 272. (Russian)

[BABE] E. Barletta and E. Bedford, Existence of proper mappings from domains in $\mathbb{C}^{2}$, Indiana Univ. Math. J. 39 (1990), 315-338.

[BU] D. Burns, $A$ multi-valued Hartogs theorem and developing maps, preprint.

[BSW] D. Burns, S. Shnider, and R. Wells, On deformations of strictly pseudoconvex domains, Invent. Math. 46 (1978), 237-253.

[CHM] S. S. Chern and J. Moser, Real hypersurfaces in complex manifolds, Acta Math. 133 (1975), 219-271.

[FEF] C. Fefferman, The Bergman kernel and biholomorphic mappings of pseudoconvex domains, Invent. Math. 26 (1974), 1-65.

[FOR] J. Fornæss, Strictly pseudoconvex domains in convex domains, Amer. J. Math. 98 (1976), 529-569.

[GAV] E. Gavosto, Thesis, Washington University, 1990.

[GRA] I. Graham, Boundary behavior of the Caratheodory and Kobayashi metrics on strongly pseudoconvex domains in $\mathbb{C}^{n}$ with smooth boundary, Trans. Amer. Math. Soc. 207 (1975), 219-240.

[GK1] R. E. Greene and S. G. Krantz, Stability properties of the Bergman kernel and curvature properties of bounded domains, Recent Progress in Several Complex Variables, Princeton Univ. Press, Princeton, NJ, 1982.

[GK2] _ Deformation of complex structures, estimates for the $\bar{\partial}$ equation, and stability of the Bergman kernel, Adv. Math. 43 (1982), 1-86.

[GK3] _ Methods for studying the automorphism groups of weakly pseudoconvex domains, Proc. Internat. Conf. on Complex Geometry (Cetraro, Italy), Mediterranean Press, Calabria, 1991.

[HU1] X. Huang, Some applications of Bell's theorem to weakly pseudoconvex domains, Pacific J. Math. (to appear).

[HU2] __ A boundary rigidity problem of holomorphic mappings on weakly pseudoconvex domains, preprint.

[HU3] _ Preservation principle of extremal mappings and its applications, Illinois J. Math. (to appear).

[KAT] Y. Katznelson, An introduction to harmonic analysis, Dover, New York, 1976. 
[KOB] S. Kobayashi, Hyperbolic manifolds and holomorphic mappings, Marcel Dekker, New York, 1970.

[KR1] S. G. Krantz, Function theory of several complex variables, Wiley, New York, 1982.

[KR2] _ A new compactness principle in complex analysis, Univ. Autonoma de Madrid, 1987.

[KOR] J. Kohn and H. Rossi, On the extension of holomorphic functions from the boundary of a complex manifold, Ann. of Math. (2) 81 (1965), 451-472.

[KRV] N. Kruzhilin and A. Vitushkin, Extension of local mappings of pseudoconvex surfaces, Dokl. Akad. Nauk. SSSR 270 (1983), 271-274.

[LĖM1] L. Lempert, La metrique Kobayashi et les representation des domains sur la boule, Bull. Soc. Math. France 109 (1981), 427-474.

[LEM2] _ Holomorphic retracts and intrinsic metrics in convex domains, Anal. Math. 8 (1982), 257-261.

[LEM3] _ Intrinsic distances and holomorphic retracts, Complex Analysis and Applications, Sofia, 1984.

[PIN] S. Pincuk, On the analytic continuation of holomorphic mappings, Mat. USSR-Sb. 27 (1975), 416-435.

[RU1] W. Rudin, Holomorphic maps that extend to automorphisms of a ball, Proc. Amer. Math. Soc. 81 (1981), 429-432.

[RU2] _ Function theory of the unit ball in $\mathbb{C}^{n}$, Springer-Verlag, Berlin and New York, 1980.

[SIB] N. Sibony, Unpublished notes.

[VEL] J. Velling, Thesis, Stanford, 1985.

[VIT] A. G. Vitushkin, Real analytic hypersurfaces of complex manifolds, Uspekhi Mat. Nauk. 40 (1985), 3-31.

[WOG] W. Wogen, Composition operators acting on spaces of holomorphic functions on domains in $\mathbb{C}^{n}$, Operator Theory: Operator Algebras and Applications, Part 2 (Durham, New Hampshire, 1988), Proc. Sympos. Pure Math., vol. 51, Part 2, Amer. Math. Soc., Providence, RI, 1990, pp. 361-366.

[WU] H. H. Wu, Normal families of holomorphic mappings, Acta Math. 119 (1967), 193-2333.

[YAU] S. T. Yau, A generalized Schwarz lemma for Kähler manifolds, Amer. J. Math. 100 (1978), 197-204.

ABSTRACT. A rigidity theorem for holomorphic mappings, in the nature of the uniqueness statement of the classical one-variable Schwarz lemma, is proved at the boundary of a strongly pseudoconvex domain. The result reduces to an interesting, and apparently new, result even in one complex dimension. The theorem has a variety of geometric and analytic interpretations.

Department of Mathematics, University of Michigan, ANN Arbor, Michigan 48109

Department of Mathematics, BoX 1146, Washington University in St. Louis, ST. Louis, MISSOURI 63130 